\title{
RELIABLE NUMERICAL MODELLING OF MALARIA PROPAGATION
}

\author{
István Faragó, Miklós Emil Mincsovics, Rahele Mosleh, Budapest
}

Received April 3, 2018. Published online June 25, 2018.

\begin{abstract}
We investigate biological processes, particularly the propagation of malaria. Both the continuous and the numerical models on some fixed mesh should preserve the basic qualitative properties of the original phenomenon. Our main goal is to give the conditions for the discrete (numerical) models of the malaria phenomena under which they possess some given qualitative property, namely, to be between zero and one. The conditions which guarantee this requirement are related to the time-discretization step-size. We give a sufficient condition for some explicit methods. For implicit methods we prove that the above property holds unconditionally.
\end{abstract}

Keywords: epidemic model; qualitative propertie; non-negativity; finite difference method

MSC 2010: 65M06, 35Q92, 34C60

\section{INTRODUCTION}

Mathematical models are efficient tools of modelling different phenomena. In order to have an adequate model, it is almost obvious that the continuous model and on some fixed mesh the numerical model should preserve the basic qualitative properties of the original phenomenon. Such models are called qualitatively adequate, or, in short, reliable models.

In some earlier works, we have investigated discrete models of the heat conduction problem (e.g. [4], [5]). For the heat conduction process the main and physically motivated characteristic properties are the non-negativity preservation, the maximum/minimum principle and the contractivity in the maximum norm. In biology

The authors were supported by the Hungarian Research Fund OTKA under grant no. K112157 and SNN-125119. 
one of the most challenging problems is the investigation of epidemic propagation. The mathematical/numerical modelling of epidemic propagation may reveal valuable information about the main characteristics of the epidemic under study.

This is why a number of mathematical models have been constructed and investigated in the literature, see e.g. [2]. The investigations started almost one hundred years ago with a model based on a system of ordinary differential equations, given in [7]. This model is a so-called compartmental model, where the population is divided into disjoint groups according to the members' relation to the disease, and the timedependent function of the number of the members in each group is determined by solving a system of ordinary differential equations. The most typical compartments are as follows: susceptibles (members that can be infected), infectives (members that can pass on the disease to others) and recovered (members that have recovered from the disease). The basic models are the SIR-type models and their modifications. When the birth and the death of the members are not taken into account, then the main qualitative properties of such a disease propagation process include mass conservation, non-negativity preservation and monotonicity of the numbers of the susceptibles and the recovered members.

One of the most current epidemic spreading issues today is to investigate the spread of malaria.

Malaria is a parasitic vector borne disease endemic in many parts of the world. The disease is a killer and as global warming occurs, endemic malaria will spread to more areas. In 2016, nearly half of the world's population was at risk of malaria. Most of malaria's cases and deaths occur in sub-Saharan Africa. However, WHO regions South-East Asia, Eastern Mediterranean, Western Pacific, the United States, Europe (especially Romania, Italy, Greece, Hungary and Austria) are also at risk [12], [3].

The paper is organized as follows. In Section 2 we describe the continuous models for malaria and investigate the required qualitative property for the models. In Section 3 we define and analyze the discretized Ross and delayed Ross-Macdonald models. In Section 4 we give numerical examples for the discrete models. Finally, in Section 5 we conclude the paper.

\section{Continuous models of MALARia}

A mathematical model describes the dynamics of malaria and human population compartments. There are different mathematical models of this disease. This paper will examine mainly the Ross model and its delayed modification in order to achieve a greater understanding of this phenomenon. Malaria parasite is transmitted from person to person by a female Anopheles mosquito. The symptoms of malaria of 
an infected human include bouts of fever and anemia. On average, the incubation period is about 12 days in humans and about 10 days in mosquitoes.

The amount of literature for the mathematical modelling of this disease is increasing rapidly. (See e.g. [6], [8] and the references within.) In most of these models, usually the following assumptions are made.

$\triangleright$ Total populations are constant for each groups.

$\triangleright$ The infection in the human confers negligible immunity and does not result in death or isolation.

$\triangleright$ All newborns are susceptible.

$\triangleright$ Susceptible humans can receive the infection only by contacting with infective mosquitoes.

$\triangleright$ A susceptible mosquito can receive the infection only from an infectious human.

2.1. The Ross model. The connection between mosquitoes and malaria was first observed by Sir Ronald Ross [9]. In the construction of the model, he made several assumptions in order to simplify and solve his equations. (E.g. for humans the death rate was negligible compared with the recovery rate and the opposite assumption holds for the mosquitoes, the birth rate of the mosquito equals its death rate.) In this model the groups of humans and mosquitoes are divided into two subgroups, namely, infected and susceptible ones. Hence, denoting by $x(t)$ and $y(t)$ the density of infected humans and mosquitoes, respectively, the Ross model has the form

$$
\begin{aligned}
& \dot{x}(t)=\alpha y(t)(1-x(t))-r x(t), \\
& \dot{y}(t)=\beta x(t)(1-y(t))-\mu y(t) .
\end{aligned}
$$

The positive parameters $\alpha, \beta, r$ and $\mu$ in (1) are computed from the available biological information, like biting rate, proportion of bites that produce infection in humans, proportion of bites by which one susceptible mosquito becomes infected, etc. We note that the functions $x(t)$ and $y(t)$ could also be viewed as the number of infected humans and infected mosquitoes, under the assumption that the total populations, which are constant for each group, are equal to one.

Hence, supplied with the initial values $x(0)$ and $y(0)$, the Ross model (1) yields a Cauchy problem for a system of two unknown functions.

Clearly, $x(0)$ and $y(0)$ are between zero and one. However, this property should be preserved for any $t \geqslant 0$. Hence, it is quite natural to require that for any time instant, starting from a suitable initial condition, the solutions of (1) have the above property. In what follows, we will refer to this property as density preservation (DP) property. 
In the following we examine this property.

Theorem 1. The Ross model (1) has the DP property.

P r o of. We have to show that for any $x(0), y(0) \in[0,1]$ the relation $x(t), y(t) \in$ $[0,1]$ is valid for all $t>0$. Using the notation

$$
\Omega=\{(x, y): 0 \leqslant x, y \leqslant 1\}
$$

we prove that the set $\Omega$ is positively invariant with respect to the Ross model (1). To this aim we should show that the trajectories have to be reflected back when they reach the boundary of $\Omega$. If $\bar{t}$ denotes the time-instant when the trajectory reaches the boundary, we only have to check the sign of the derivatives at the boundary at this time-point. Since

$$
\begin{array}{lllll}
\dot{x}(\bar{t})=\alpha y(\bar{t}) \geqslant 0 & \text { if } x(\bar{t})=0 & \text { and } & \dot{x}(\bar{t})=-r<0 & \text { if } x(\bar{t})=1, \\
\dot{y}(\bar{t})=\beta x(\bar{t}) \geqslant 0 & \text { if } y(\bar{t})=0 & \text { and } & \dot{y}(\bar{t})=-\mu<0 & \text { if } y(\bar{t})=1,
\end{array}
$$

the statement is proven.

The feedback dynamics from mosquito to human and back to mosquito involve considerable time delays due to the incubation periods of the parasites. This leads to another model.

2.2. Delayed Ross-Macdonald model. Some modellers have noticed the omission of latencies in the Ross model and proposed modifications in the form of delayed differential equations. However, most of these works only incorporate a single delay, representing the latency of the parasite in mosquitoes. Ruan and Xiao modified the Ross model by adding two delays accounting for the latencies in mosquitoes and humans, respectively [10]. In this model for the transmission of the pathogen it is assumed that the human and the mosquitoes remain exposed for some time (which is called delay) and then become infectious. The delays are denoted by $\tau_{h}$ (latent period for human) and $\tau_{m}$ (latent period for mosquito). Obviously they are assumed to be non-negative. The delays may be just constants (the so-called constant delay case) or functions of time (time-dependent delay case). In the delayed Ross-Macdonald model the constant delay is given and has the form

$$
\begin{aligned}
& \dot{x}(t)=\alpha y\left(t-\tau_{h}\right)\left(1-x\left(t-\tau_{h}\right)\right) \mathrm{e}^{-r \tau_{h}}-r x(t), \\
& \dot{y}(t)=\beta x\left(t-\tau_{m}\right)\left(1-y\left(t-\tau_{m}\right)\right) \mathrm{e}^{-\mu \tau_{m}}-\mu y(t) .
\end{aligned}
$$


The terms $\mathrm{e}^{-r \tau_{h}}$ and $\mathrm{e}^{-\mu \tau_{m}}$ account for the probability that an infected human host (mosquito) can survive the latent period $\tau_{h}$ (and $\tau_{m}$ respectively). This model assumed the knowledge of the unknown functions $x(t)$ and $y(t)$ on the interval $\left[-\tau^{\star}, 0\right]$, where $\tau^{\star}=\max \left\{\tau_{h}, \tau_{m}\right\}$; they are also called history functions. The following statement deals with a qualitative property of the above model.

Theorem 2. Let the history functions be mappings of type $\left[-\tau^{\star}, 0\right] \longmapsto[0,1]$, and assume that the conditions

$$
\alpha \mathrm{e}^{-r \tau_{h}}-r \leqslant 0 \text { and } \beta \mathrm{e}^{-\mu \tau_{m}}-\mu \leqslant 0
$$

hold. Then the delayed Ross-Macdonald model (3) possesses the DP property.

P r o of. Delay differential equations of this type can be handled as ODEs using the method of steps, thus positive invariance of the set $\Omega$ can be treated similarly. (Cf. [11], Theorem 3.4.) This means that we have to check the derivatives at the boundary of the set. We denote again the time-instant when the trajectory reaches the boundary by $\bar{t}$. Since

$$
\begin{aligned}
& \dot{x}(\bar{t})=\alpha y\left(\bar{t}-\tau_{h}\right)\left(1-x\left(\bar{t}-\tau_{h}\right)\right) \mathrm{e}^{-r \tau_{h}} \geqslant 0 \quad \text { if } x(\bar{t})=0, \\
& \dot{x}(\bar{t})=\alpha y\left(\bar{t}-\tau_{h}\right)\left(1-x\left(\bar{t}-\tau_{h}\right)\right) \mathrm{e}^{-r \tau_{h}}-r \leqslant \alpha \mathrm{e}^{-r \tau_{h}}-r \leqslant 0 \quad \text { if } x(\bar{t})=1, \\
& \dot{y}(\bar{t})=\beta x\left(\bar{t}-\tau_{m}\right)\left(1-y\left(\bar{t}-\tau_{m}\right)\right) \mathrm{e}^{-\mu \tau_{m}} \geqslant 0 \quad \text { if } y(\bar{t})=0, \\
& \dot{y}(\bar{t})=\beta x\left(\bar{t}-\tau_{m}\right)\left(1-y\left(\bar{t}-\tau_{m}\right)\right) \mathrm{e}^{-\mu \tau_{m}}-\mu \leqslant \beta \mathrm{e}^{-\mu \tau_{m}}-\mu \leqslant 0 \quad \text { if } y(\bar{t})=1,
\end{aligned}
$$

the statement is proven.

We note that without the condition (4) we cannot guarantee that the solution will not exceed the upper bound.

\section{Numerical METHODS FOR CONTINUOUS MALARIA MODELS}

We are interested to know whether the positive invariance of the set $\Omega$ is preserved on the discrete level. Naturally, a numerical method can be considered applicable if this property is preserved. In some case this holds unconditionally, while in other cases we have to impose restrictions for the discretization step-size $\Delta t$. In the sequel, we investigate the explicit Euler and implicit Euler discretization methods for the continuous problems, formulated in the previous part.

3.1. Explicit Euler method applied to the Ross model. First we apply the simplest explicit discretization method, the explicit Euler method to the Ross 
model (1), which results in the discrete model:

$$
\begin{aligned}
& x_{n+1}=x_{n}+\Delta t\left(\alpha y_{n}\left(1-x_{n}\right)-r x_{n}\right), \\
& y_{n+1}=y_{n}+\Delta t\left(\beta x_{n}\left(1-y_{n}\right)-\mu y_{n}\right) .
\end{aligned}
$$

We have to show the positive invariance of the set $\Omega$ of this method. Therefore, we need to guarantee that for any $x_{n}, y_{n} \in[0,1]$ the discrete model (5) results in $x_{n+1}$, $y_{n+1} \in[0,1]$.

Obviously, $0 \leqslant x_{n+1}$ means that $x_{n} \geqslant \Delta t\left(-\alpha y_{n}\left(1-x_{n}\right)+r x_{n}\right)$. If

$$
-\alpha y_{n}\left(1-x_{n}\right)+r x_{n} \leqslant 0
$$

then this condition is satisfied for any $\Delta t$, without restriction. Otherwise, we have a condition for the step-size as follows:

$$
\Delta t \leqslant \frac{x_{n}}{-\alpha y_{n}\left(1-x_{n}\right)+r x_{n}} .
$$

Hence, due to the inequality

$$
\frac{1}{r} \leqslant \frac{x_{n}}{-\alpha y_{n}\left(1-x_{n}\right)+r x_{n}}
$$

we get that the choice of $\Delta t$ as $\Delta t \leqslant 1 / r$ ensures the non-negativity of $x_{n+1}$. Similarly, we can guarantee the non-negativity of $y_{n+1}$ by choosing $\Delta t$ such that $\Delta t \leqslant 1 / \mu$.

The upper bound for $x_{n+1}$, i.e., the condition $x_{n+1} \leqslant 1$ means that $1-x_{n} \geqslant$ $\Delta t\left(\alpha y_{n}\left(1-x_{n}\right)-r x_{n}\right)$.

If

$$
\alpha y_{n}\left(1-x_{n}\right)-r x_{n} \leqslant 0
$$

then this condition is satisfied for any $\Delta t$, without restriction. Otherwise, we have the condition

$$
\Delta t \leqslant \frac{1}{\alpha} \leqslant \frac{1-x_{n}}{\alpha y_{n}\left(1-x_{n}\right)-r x_{n}},
$$

with the consequence that the property $x_{n+1} \leqslant 1$ is satisfied under the assumption $\Delta t \leqslant 1 / \alpha$. Similarly, for the property $y_{n+1} \leqslant 1$ we assume that $\Delta t \leqslant 1 / \beta$.

We summarize this result in the following theorem.

\section{Theorem 3. If}

$$
\Delta t \leqslant \min \left\{\frac{1}{r}, \frac{1}{\mu}, \frac{1}{\alpha}, \frac{1}{\beta}\right\}
$$

then the explicit Euler method applied to the Ross model (5) possesses the DP property. 
Re m a r k 1. We could see that under the conditions (6) and (7) for the explicit scheme the bound (8) can be omitted. This means that on a uniform mesh these conditions should be satisfied for any $x_{n}$ and $y_{n}$. This raises the question of whether this condition can be guaranteed a priori. That means if the condition is true for some $x_{0}$ and $y_{0}$, then is it also fulfilled for $x_{1}$ and $y_{1}$, defined by (1)? It is easy to check in some concrete example that it is not so.

3.2. Implicit Euler method applied to the Ross model. Hereinafter for the discretization of the continuous Ross model (1) we apply the implicit Euler method, which results in the discrete model

$$
\begin{aligned}
& x_{n+1}=x_{n}+\Delta t\left(\alpha y_{n+1}\left(1-x_{n+1}\right)-r x_{n+1}\right), \\
& y_{n+1}=y_{n}+\Delta t\left(\beta x_{n+1}\left(1-y_{n+1}\right)-\mu y_{n+1}\right) .
\end{aligned}
$$

The positive invariance of the set $\Omega$ means again that we need to guarantee that $x_{n+1}, y_{n+1} \in[0,1]$ if $x_{n}, y_{n} \in[0,1]$.

From (9) we get that $y_{n+1}$ can be expressed as

$$
y_{n+1}=p x_{n+1}+q_{n}
$$

where

$$
p=\frac{\beta(1+\Delta t r+\Delta t \alpha)}{\alpha(1+\Delta t \beta+\Delta t \mu)}, \quad q_{n}=\frac{\alpha y_{n}-\beta x_{n}}{\alpha(1+\Delta t \beta+\Delta t \mu)} .
$$

Substituting this expression into the first equation of (9) we obtain the equation for the $x_{n+1}$ as follows

$$
A x_{n+1}^{2}+B_{n} x_{n+1}+C_{n}=0,
$$

which is a quadratic algebraic equation with the coefficients

$$
A=-\Delta t \alpha p<0, \quad B_{n}=\Delta t \alpha\left(p-q_{n}\right)-\Delta t r-1,
$$

and

$$
C_{n}=x_{n}+\Delta t \alpha q_{n}=\frac{x_{n}+\Delta t \mu x_{n}+\Delta t \alpha y_{n}}{1+\Delta t \beta+\Delta t \mu} \geqslant 0 \text {. }
$$

Since the discriminant is non-negative, the two roots $x_{n+1}^{(1)}, x_{n+1}^{(2)}$ of (11) are real numbers. Moreover, for their product we have the obvious relation

$$
x_{n+1}^{(1)} x_{n+1}^{(2)}=\frac{C_{n}}{A} \leqslant 0 .
$$


Note that the case $C_{n}=0$ means that $x_{n}=y_{n}=0$. In this case the two roots are $x_{n+1}=0$ and $x_{n+1}=-B / A$. Because $x(0)=y(0)=0$ is an equilibrium point of the Ross system, it is quite natural to choose $x_{n+1}=0$. Otherwise we have one negative and one positive root, which will be denoted as $x_{n+1}^{-}$and $x_{n+1}^{+}$, respectively. For these roots we have the relation

$$
\frac{x_{n+1}^{-}+x_{n+1}^{+}}{2}=-\frac{B_{n}}{2 A}=\frac{\alpha\left(p-q_{n}\right)-r}{2 \alpha p}-\frac{1}{2 \alpha p \Delta t} .
$$

We notice that the parameters $p$ and $q_{n}$ in (10) depend on $\Delta t$ and they are bounded as $\Delta t \rightarrow 0$. Hence, letting $\Delta t \rightarrow 0$ the first expression on the right-hand side of (12) is bounded, while the second part tends to $-\infty$. This means that

$$
\left|x_{n}-x_{n+1}^{-}\right| \rightarrow \infty
$$

with the consequence that we choose the positive root to get the approximation for $x\left(t_{n+1}\right)$, i.e., we put $x_{n+1}=x_{n+1}^{+}$. Hence, the non-negativity of $x_{n+1}$ is ensured.

Using the second equation of (9), we can see that the non-negativity of $x_{n+1}$ implies the non-negativity of $y_{n+1}$, too.

Now, by using the first equation in (9), we have

$$
x_{n+1}\left(1+\alpha \Delta t y_{n+1}+r \Delta t\right)=x_{n}+\alpha \Delta t y_{n+1} .
$$

From this relation we can see that if $y_{n+1} \geqslant 0$, then $0 \leqslant x_{n+1}<1$. Similarly, under the condition $x_{n+1} \geqslant 0$ the relation $0 \leqslant y_{n+1}<1$ is also valid. This completes the argument, since the previous item shows that $x_{n+1}, y_{n+1} \geqslant 0$ holds unconditionally.

We can summarize the result in the following theorem.

Theorem 4. The implicit Euler method applied to the Ross model (9) possesses the DP property unconditionally.

\subsection{Explicit Euler method applied to the delayed Ross-Macdonald model.}

We apply the explicit Euler method to the Ross-Macdonald model (3), which results in the discrete model

$$
\left\{\begin{array}{l}
x_{n+1}=x_{n}+\Delta t\left(\alpha y_{n, \tau_{h}}\left(1-x_{n, \tau_{h}}\right) \mathrm{e}^{-r \tau_{h}}-r x_{n}\right), \\
y_{n+1}=y_{n}+\Delta t\left(\beta x_{n, \tau_{m}}\left(1-y_{n, \tau_{m}}\right) \mathrm{e}^{-r \tau_{m}}-\mu y_{n}\right) .
\end{array}\right.
$$

where, e.g., $x_{n, \tau_{h}}$ approximates the value $x\left(t_{n}-\tau_{h}\right)$. The value $t_{n}-\tau_{h}$ is not necessarily a grid-point, thus we have to use interpolation. For first order methods 
a linear interpolation is reasonable, see e.g. [1], Section 3.1, the Feldstein method, which means that $x_{n, \tau_{h}}$ is taken as a convex combination of the known $x_{j}$ and $x_{j+1}$, where $t_{j} \leqslant t_{n}-\tau_{h} \leqslant t_{j+1}$.

Recalling that the continuous model possessed the DP property under the assumption (4), our goal is to guarantee the DP property on the discrete level under the same assumption.

First, we notice that the calculation of $0 \leqslant x_{n+1}$ goes exactly in the same way as for the non-delayed case, and leads to the same bound as there.

The inequality $x_{n+1} \leqslant 1$ means that $1-x_{n} \geqslant \Delta t\left(\alpha y_{n, \tau_{h}}\left(1-x_{n, \tau_{h}}\right) \mathrm{e}^{-r \tau_{h}}-r x_{n}\right)$. If the right-hand side is non-positive, then it is satisfied.

Note that the function

$$
g(x)=\frac{1-x}{\alpha \mathrm{e}^{-r \tau_{h}}-r x}
$$

is monotonically increasing, since

$$
g^{\prime}(x)=\frac{-\alpha \mathrm{e}^{-r \tau_{h}}+r}{\left(\alpha \mathrm{e}^{-r \tau_{h}}-r x\right)^{2}} \geqslant 0,
$$

thus if the right-hand side $\Delta t\left(\alpha y_{n, \tau_{h}}\left(1-x_{n, \tau_{h}}\right) \mathrm{e}^{-r \tau_{h}}-r x_{n}\right)$ is positive, then

$$
\Delta t \leqslant \frac{\mathrm{e}^{r \tau_{h}}}{\alpha} \leqslant \frac{1-x_{n}}{\alpha \mathrm{e}^{-r \tau_{h}}-r x_{n}} \leqslant \frac{1-x_{n}}{\alpha y_{n, \tau_{h}}\left(1-x_{n, \tau_{h}}\right) \mathrm{e}^{-r \tau_{h}}-r x_{n}} .
$$

This means that the non-negativity of $x_{n+1}$ and $y_{n+1}$ is preserved under the assumptions $\Delta t \leqslant \mathrm{e}^{r \tau_{h}} / \alpha$ and $\Delta t \leqslant \mathrm{e}^{\mu \tau_{m}} / \beta$, respectively.

We summarize this result in the following theorem.

Theorem 5. We assume that (4) holds. If

$$
\Delta t \leqslant \min \left\{\frac{1}{r}, \frac{1}{\mu}, \frac{\mathrm{e}^{r \tau_{h}}}{\alpha}, \frac{\mathrm{e}^{\mu \tau_{m}}}{\beta}\right\}
$$

then the explicit Euler method applied to the delayed Macdonald-Ross model possesses the DP property.

\subsection{Implicit Euler method applied to the delayed Ross-Macdonald} model. We apply the implicit Euler method to the Ross-Macdonald model (3), which results in the discrete model

$$
\left\{\begin{array}{l}
x_{n+1}=x_{n}+\Delta t\left(\alpha y_{n+1, \tau_{h}}\left(1-x_{n+1, \tau_{h}}\right) \mathrm{e}^{-r \tau_{h}}-r x_{n+1}\right), \\
y_{n+1}=y_{n}+\Delta t\left(\beta x_{n+1, \tau_{m}}\left(1-y_{n+1, \tau_{m}}\right) \mathrm{e}^{-r \tau_{m}}-\mu y_{n+1}\right) .
\end{array}\right.
$$


We assume that the step-size is small compared to the delays, i.e.,

$$
\Delta t \leqslant \min \left\{\tau_{h}, \tau_{m}\right\}
$$

and we assume the natural condition (4) as well. In this case $x_{n+1, \tau_{h}}$ is a convex combination of two already known values $x_{j}$ and $x_{j+1}, j<n$ and we can handle $y_{n+1, \tau_{h}}$ similarly.

Using the form $x_{n+1}(1+\Delta t r)=x_{n}+\Delta t \alpha \mathrm{e}^{-r \tau_{h}} y_{n+1, \tau_{h}}\left(1-x_{n+1, \tau_{h}}\right)$, we can see that $x_{n+1} \geqslant 0$. Moreover,

$$
x_{n+1}=\frac{x_{n}+\Delta t \alpha \mathrm{e}^{-r \tau_{h}} y_{n+1, \tau_{h}}\left(1-x_{n+1, \tau_{h}}\right)}{1+\Delta t r} \leqslant \frac{1+\Delta t \alpha \mathrm{e}^{-r \tau_{h}}}{1+\Delta t r} \leqslant 1 .
$$

We summarize this result in the following theorem.

Theorem 6. We assume that (4) holds. If (16) holds, then the implicit Euler method applied to the delayed Macdonald-Ross model possesses the DP property.

\section{NumERICAL EXPERIMENTS}

For the explicit scheme (5) and for the implicit scheme (9) we check our theoretical result, given in Theorem 3 on examples and we also examine the sharpness of the theoretical bound (8) of the explicit scheme. The computations were done with the parameters $\alpha=0.072, \beta=0.2, r=0.002$ and $\mu=0.2$. For these parameters, condition (8) in Theorem 3 yields the bound $\Delta t \leqslant 5$. In figures star symbols stand for humans and solid lines for mosquitos.

For the initial density the values $x(0)=0.01$ and $y(0)=0.01$ were set. Figure 1 (a) shows the result for this experiment with $\Delta t=4$, which satisfies the condition (8). We can see that the solutions are between the bounds. Figure 1 (b) illustrates the result for the step-size $\Delta t=7$, which is above the condition. This picture shows that the system has exploded with this parameter.

The condition (8) is sufficient for the discrete DP property. Using the above model parameters, with the initial condition $x(0)=y(0)=0.5$, we can illustrate its sharpness, too.

In Figure 2 (a) the numerical result is shown for $\Delta t=4.9$, which is still below the bound (8). Although the result is correct, i.e., the DP property holds, apparently in the initial stage the density function of mosquitoes oscillates.

In Figure 2 (b) the numerical result is shown for $\Delta t=5.2$, which is above the bound (8). As we can see, even for this bigger parameter the model is DP preserving 


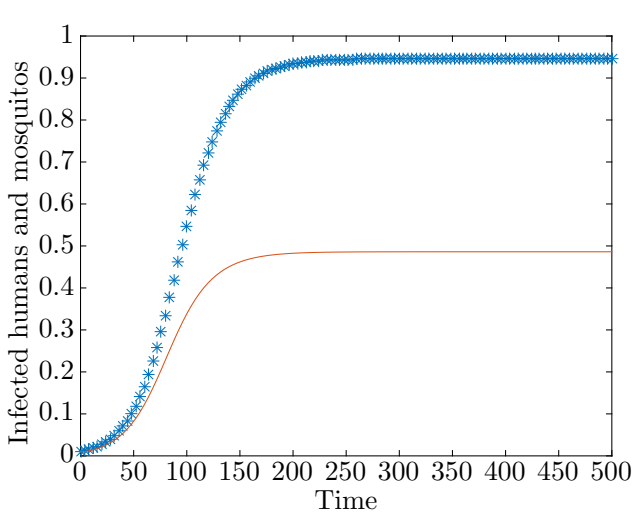

(a) $\Delta t=4$

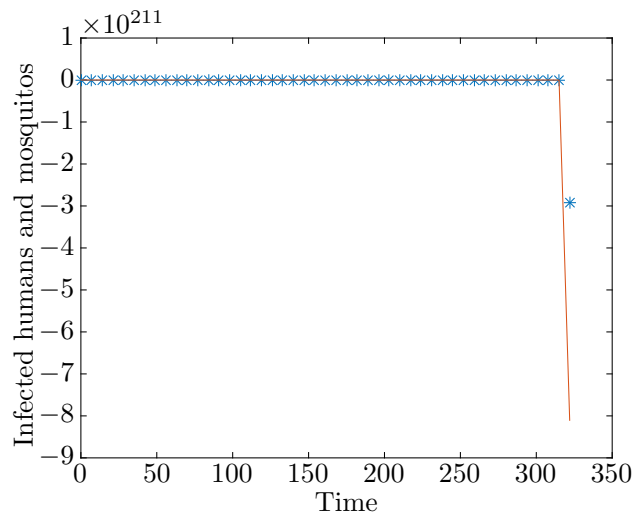

(b) $\Delta t=7$

Figure 1. Explicit scheme with the step-size $\Delta t$ where the condition (8) is (a) satisfied, (b) violated.

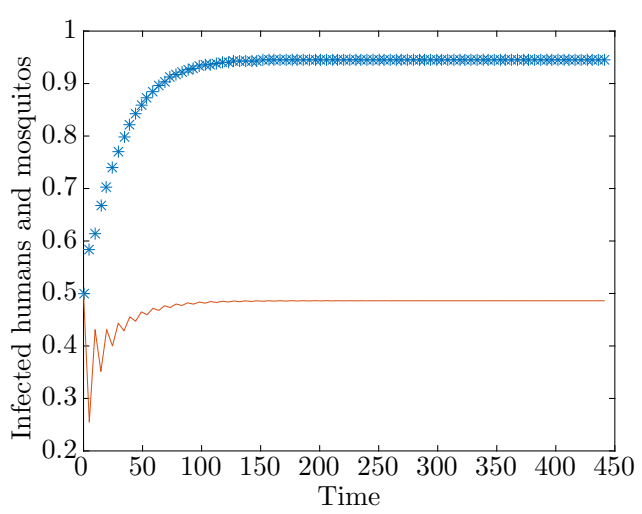

(a) $\Delta t=4.9$

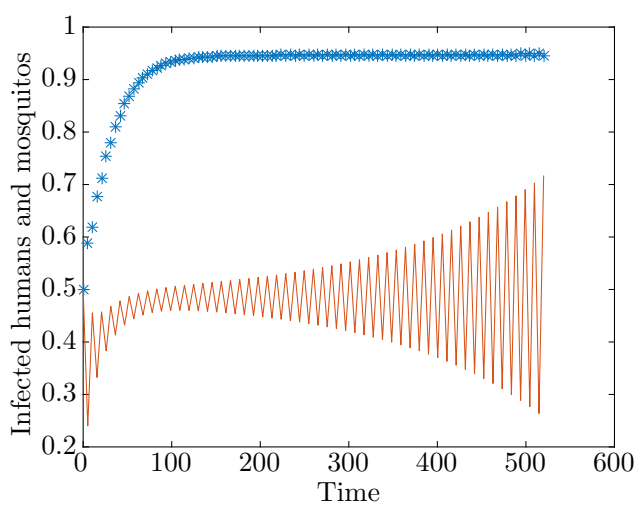

(b) $\Delta t=5.2$

Figure 2. Explicit scheme with the step-size $\Delta t$ where the condition (8) is (a) satisfied, (b) violated.

on the considered interval, which shows that the bound (8) is a sufficient, but not necessary condition for the DP property. However, we note that in the whole stage the density function of mosquitoes strongly oscillates.

If we put a bit bigger $\Delta t$, namely, $\Delta t=5.3$, then the model loses this property, the DP property is no longer valid. (See Figure 3 (a).) However, for the implicit Euler method, which has no bound for $\Delta t$, we obtain correct results, as is shown in Figure 4 (a).

If we choose quite a big $\Delta t$, e.g., $\Delta t=8$, the model will continue to preserve the DP property (see Figure 4 (b)) but not for the explicit scheme (see Figure 3 (b)). 


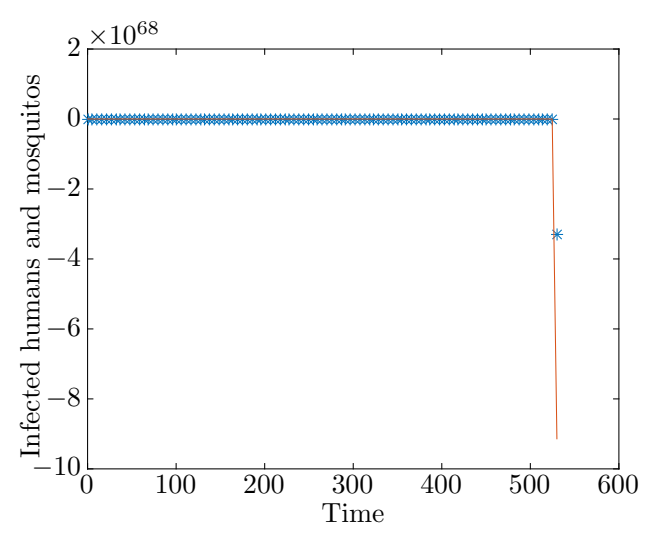

(a) $\Delta t=5.3$

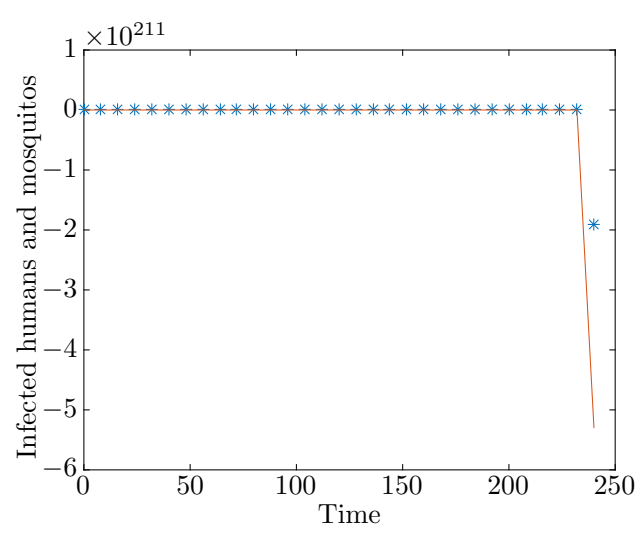

(b) $\Delta t=8$

Figure 3. Explicit scheme with the step-size $\Delta t$ where the condition (8) is violated.

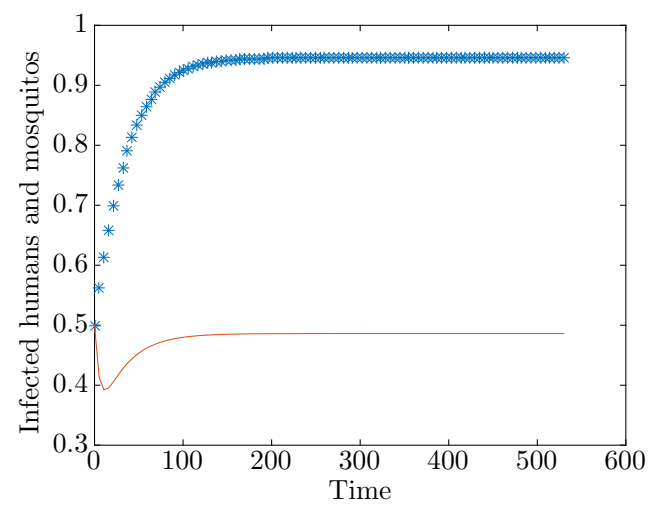

(a) $\Delta t=5.3$

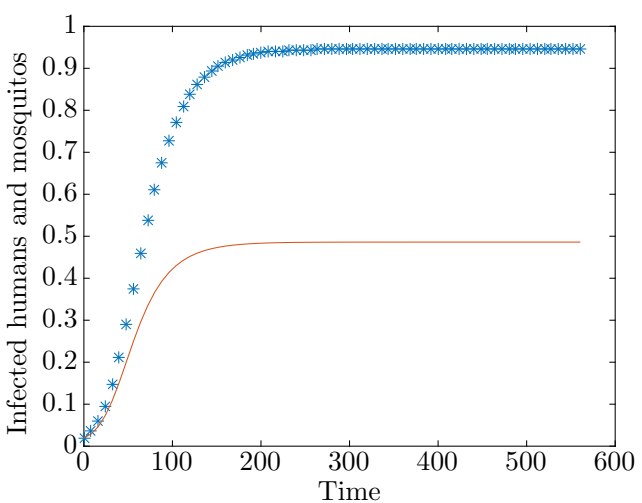

(b) $\Delta t=8$

Figure 4. Implicit scheme with the step-size $\Delta t$ (there is no condition).

\section{Summary}

In this paper we have investigated mathematically the propagation of malaria. We gave two basic models, the Ross model and the delayed Ross-Macdonald model. Both the continuous and the numerical models on some fixed mesh should preserve a basic qualitative property of the original phenomenon, which is the DP property. The continuous Ross model possesses this property. For the discrete Ross model we gave a sufficient condition for explicit schemes. However, for the implicit scheme this property holds unconditionally. For the continuous delayed Ross-Macdonald model the DP property holds conditionally. We also formulated those conditions under 
which the discrete models (both explicit and implicit) have this property. Numerical examples are given which verify the theoretical results.

\section{References}

[1] A. Bellen, M. Zennaro: Numerical Methods for Delay Differential Equations. Numerical Mathematics and Scientific Computation, Oxford University Press, Oxford, 2003.

[2] V. Capasso: Mathematical Structures of Epidemic Systems. Lecture Notes in Biomathematics 97, Springer, Berlin, 2008.

[3] ECDC: Communicable Disease Threats Report Week 46, 12-18 November 2017. Available at: https://ecdc.europa.eu/en/publications-data/communicable-diseasethreats-report-12-18-november-2017-week-46.

[4] I. Faragó, R. Horváth: Discrete maximum principle and adequate discretizations of linear parabolic problems. SIAM J. Sci. Comput. 28 (2006), 2313-2336.

[5] I. Faragó, R. Horváth: Continuous and discrete parabolic operators and their qualitative properties. IMA J. Numer. Anal. 29 (2009), 606-631.

[6] H. W. Hethcote, H.W. Stech, P. van den Driessche: Periodicity and stability in epidemic models: a survey. Differential Equations and Applications in Ecology, Epidemics, and Population Problems (S. Busenberg, K. L. Cooke, eds.). Academic Press, New York, 1981, pp. 65-82.

[7] W. O.Kermack, A. G. McKendrick: A contribution to the mathematical theory of epidemics. Proc. R. Soc. Lond., Ser. A 115 (1927), 700-721.

8] S. Mandal, R. R. Sarkar, S. Sinha: Mathematical models of malaria-a review. Malaria Journal 10 (2011).

[9] R. Ross: The Prevention of Malaria. John Murray, London, 1911.

[10] S. Ruan, D. Xiao, J. C. Beier: On the delayed Ross-Macdonald model for malaria transmission. Bull. Math. Biol. 70 (2008), 1098-1114.

[11] H. Smith: An Introduction to Delay Differential Equations with Applications to the Life Sciences. Texts in Applied Mathematics 57, Springer, New York, 2011.

[12] WHO.malaria: Available at: http://www.who.int/en/news-room/fact-sheets/detail/malaria.

Authors' addresses: István Faragó, Department of Applied Analysis and Computational Mathematics, Faculty of Science, Eötvös Loránd University, 1117, Budapest Pazmany P. s. 1/C., Hungary; MTA-ELTE Research Group, 1117, Budapest Pazmany P. s. 1/C., Hungary; Department of Differential Equations, Budapest University of Technology and Economics, 1111 Budapest Egry József str. 1, Hungary, e-mail: faragois@cs.elte.hu; Miklós Emil Mincsovics, MTA-ELTE Research Group, 1117, Budapest Pazmany P. s. 1/C., Hungary; Department of Differential Equations, Budapest University of Technology and Economics, 1111 Budapest Egry József str. 1, Hungary, e-mail: mincso@cs.elte.hu; Rahele Mosleh, Department of Differential Equations, Budapest University of Technology and Economics, 1111 Budapest Egry József str. 1, Hungary, e-mail: rmosleh028@gmail.com. 